\title{
Fast Monostatic Scattering Analysis Based on Bayesian Compressive Sensing
}

\author{
Huan-Huan Zhang ${ }^{1}$, Xun-Wang Zhao ${ }^{1}$, Zhong-Chao Lin ${ }^{1}$, and Wei E. I. Sha ${ }^{2}$ \\ ${ }^{1}$ The School of Electronic Engineering \\ Xidian University, Xi'an, 710071, China \\ zhanghuanajkd@outlook.com \\ ${ }^{2}$ The Department of Electrical and Electronic Engineering \\ The University of Hong Kong, Hong Kong, China
}

\begin{abstract}
The Bayesian compressive sensing algorithm is utilized together with the method of moments to fast analyze the monostatic electromagnetic scattering problem. Different from the traditional compressive sensing based fast monostatic scattering analysis method which cannot determine the required measurement times, the proposed method adopts the Bayesian framework to recover the underlying signal. Error bars of the signal can be obtained in the recovery procedure, which provides a means to adaptively determine the number of compressive-sensing measurements. Numerical results are given to demonstrate the accuracy and effectiveness of proposed method.
\end{abstract}

Index Terms - Bayesian compressive sensing, method of moments, monostatic, scattering.

\section{INTRODUCTION}

Electromagnetic scattering simulation has been widely applied to the area of non-cooperative radar target identification and radar imaging to get the echo signal of radar target without measurement. Among various electromagnetic scattering analysis methods, the method of moments (MoM) has drawn great interests in the past several decades [1-3]. The MoM is particularly advantageous for the analysis of exterior open-region scattering problems duo to its inherent capability for simulating unbounded domains.

When utilizing MoM to acquire echo signals from a large amount of aspect angles, one has to run the simulation code equal times with the number of aspect angles to obtain all the echo signals. For instance, to obtain the inverse synthetic aperture radar (ISAR) image of the B2 model as shown in Fig. 1, one has to get the wideband scattered field data from 720 aspect angles of the model. Actually, it takes much time to analyze the electrically large objects only once, let alone many times. So there is an urgent demand to accelerate the simulation process of monostatic scattering.

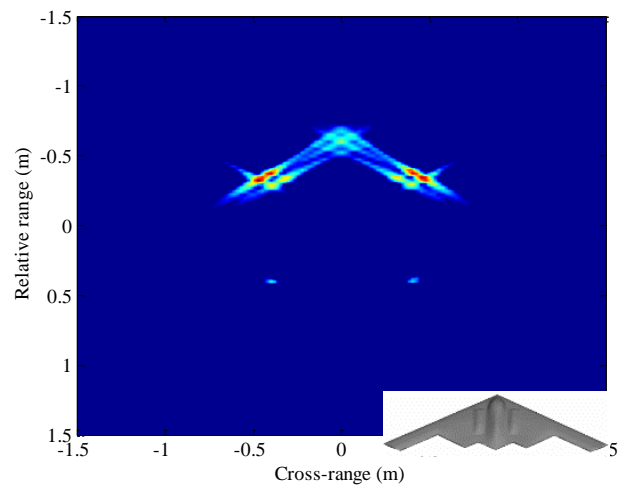

Fig. 1. Inverse synthetic aperture radar (ISAR) image of the B2 model based on the wideband scattered field data from 720 aspect angles of the target.

Two kinds of effort have been done to achieve this goal. One is to speed up the single simulation by using fast algorithms to accelerate the method of moments. The existing fast algorithms can mainly be classified into three categories: fast multipole method (FMM) [4-6], FFT-based methods [7-9], and low rank matrix based methods [10-12]. The alternative way is to reduce the total number of simulations using algorithms like asymptotic waveform evaluation (AWE) [13, 14], modelbased parameter estimation (MBPE) [15], excitation matrix compression methods [16, 17], etc. But these algorithms show some shortcomings. For AWE and MBPE, a multi-point expansion or interpolation is needed for wide-angle problems. The major technical challenge is to adaptively choose the expansion points or interpolation points. The excitation matrix compression methods compress the excitation matrix and remove redundancies in the initial excitation assembly. The considered full right-hand-side (RHS) matrix has to be stored explicitly. Moreover, a SVD-based compression is restricted to comparatively small matrices due to the high computational complexity. 
Compressive sensing (CS) is a rapidly emerging signal processing technique and has already been applied to electromagnetics [18-20]. A CS based method is proposed in [20] for fast analysis of wide-angle monostatic scattering problems, which falls into the second category fast algorithm described above. This method uses CS to construct a new set of right-hand-side vectors for MoM, where the number of constructed righthand-side vectors is much less than the original ones. But it is found that orthogonal matching pursuit (OMP) algorithm [21] is adopted to solve the CS optimization problem and the number of measurement cannot be determined adaptively, just like that the number of expansion or interpolation points is unknown in [13-15]. This sets up a limit for the practical applications of this technique.

Recently, more and more researchers focus on the study of Bayesian compressive sensing (BCS) method [22-25], which adopts the Bayesian framework to recover the underlying signals. Error bars of the signal can be obtained in the recovery procedure, leading to an effective strategy for adaptively determining the number of compressive-sensing measurements. The BCS method is used for coherent fusion of multi-band radar data from multiple spatially collocated radars in [24]. In [25], the BCS method is applied for estimation of the directions of arrival (DoAs) of narrow-band signals impinging on a linear antenna array. In this paper, we utilize the BCS for fast monostatic scattering calculation. Numerical results show the proposed method can determine the number of compressive-sensing measurements in an adaptive manner.

The rest of this paper is organized as follows. Section II describes the detailed theory and formulation of the proposed Bayesian compressive sensing based fast monostatic scattering analysis method. Section III demonstrates the accuracy and effectiveness of the proposed method through several numerical results. Section IV presents our conclusions.

\section{THEORY AND FORMULATION}

\section{A. Review of MoM for electromagnetic scattering problems}

For the analysis of electromagnetic scattering from perfect electrical conductor (PEC), the Maxwell's equations can be recast in the form of surface integral equations, including electric field integral equation (EFIE), magnetic field integral equation (MFIE) and combined field integral equations (CFIE). Take the following EFIE as an example:

$$
\begin{aligned}
\int_{s} \hat{t} \cdot\left[j \omega \mu J_{s}\left(\mathbf{r}^{\prime}\right) G\left(\mathbf{r}, \mathbf{r}^{\prime}\right)\right. \\
\left.\quad-\frac{j}{\omega \varepsilon}\left(\nabla^{\prime} \cdot J_{s}\left(\mathbf{r}^{\prime}\right)\right)\left(\nabla^{\prime} G\left(\mathbf{r}, \mathbf{r}^{\prime}\right)\right)\right] d S^{\prime}=\hat{t} \cdot \mathbf{E}^{i}(\mathbf{r}) .
\end{aligned}
$$

Here, $G\left(\mathbf{r}, \mathbf{r}^{\prime}\right)$ refers to the Green's function in free space. $\mathbf{r}$ and $\mathbf{r}^{\prime}$ denote the observation and source point locations. $\mathbf{E}^{i}(\mathbf{r})$ is the incident excitation plane wave. $\varepsilon$ and $\mu$ are the permittivity and permeability, respectively. $\omega$ is the angular frequency. $J_{s}\left(\mathbf{r}^{\prime}\right)$ is the unknown surface current. $\hat{t}$ refers to the tangential direction of the surface.

Equation (1) can be discretized by using MoM with planar Rao-Wilton-Glisson (RWG) basis functions [26]. The linear system of equations after Galerkin's testing is briefly outlined as follows:

$$
\sum_{n=1}^{N} Z_{m n} I_{n}=V_{m}, \quad \mathrm{~m}=1,2, \ldots, N,
$$

where

$$
\begin{gathered}
Z_{m n}=\int_{s} \Lambda_{m}(\mathbf{r}) \cdot \int_{s^{\prime}} j \omega \mu \Lambda_{n}\left(\mathbf{r}^{\prime}\right) G\left(\mathbf{r}, \mathbf{r}^{\prime}\right) d S^{\prime} d S \\
-\int_{s} \frac{j}{\omega \varepsilon} \nabla \cdot \Lambda_{m}(\mathbf{r}) \int_{s^{\prime}} \nabla^{\prime} \cdot \Lambda_{n}(\mathbf{r}) G\left(\mathbf{r}, \mathbf{r}^{\prime}\right) d S^{\prime} d S, \\
V_{m}=\int_{s} \Lambda_{m}(\mathbf{r}) \cdot \mathbf{E}^{i}(\mathbf{r}) d S .
\end{gathered}
$$

Here, $I_{n}$ represents the unknown current coefficients. Equation (2) can be written as:

$$
\mathbf{Z I}(\theta)=\mathbf{V}(\theta),
$$

where $\mathbf{Z}$ is the impedance matrix with its elements given in (3), $\mathbf{V}(\theta)$ is the right-hand-side vector related to the (4), $\mathbf{I}(\theta)$ is a vector containing the unknown current coefficients. Both the right-hand-side vector and the unknown current coefficients will change with the incident angle $\theta$.

\section{B. Basic principle of using CS for fast monostatic scattering analysis}

Suppose that multiple monostatic scattering problem with the incident angles $\theta_{1}, \theta_{2}, \cdots \theta_{M}$ is analyzed, then the following $M$ matrix equations need to be solved:

$$
\mathbf{Z I}\left(\theta_{i}\right)=\mathbf{V}\left(\theta_{i}\right), i=1,2, \cdots, M .
$$

Use $\mathrm{I}_{n}\left(\theta_{i}\right)$ to represent the current coefficient of the $n$-th element in $\mathbf{I}\left(\theta_{i}\right)$ and $n=1,2, \cdots, N$. Based on the CS theory, a measurement matrix $\phi=\left[c_{i j} \mid i=1,2, \cdots, M^{\prime}\right.$; $j=1,2, \cdots, M]$ with its elements i.i.d. Gaussian can be constructed. The measurement value of $\left\{I_{n}\left(\theta_{1}\right), I_{n}\left(\theta_{2}\right), \ldots, I_{n}\left(\theta_{M}\right)\right\}$ can be written as:

$$
\left(\begin{array}{c}
I_{n 1}^{C S} \\
I_{n 2}^{C S} \\
\vdots \\
I_{n M^{\prime}}^{C S}
\end{array}\right)=\left(\begin{array}{ccccc}
c_{11} & c_{12} & c_{13} & \cdots & c_{1 M} \\
c_{21} & c_{22} & c_{23} & \cdots & c_{2 M} \\
\vdots & \vdots & \vdots & \ddots & \vdots \\
c_{M^{\prime} 1} & c_{M^{\prime} 2} & c_{M^{\prime} 3} & \cdots & c_{M^{M} M}
\end{array}\right)\left(\begin{array}{c}
I_{n}\left(\theta_{1}\right) \\
I_{n}\left(\theta_{2}\right) \\
\vdots \\
\vdots \\
I_{n}\left(\theta_{M}\right)
\end{array}\right) .
$$


It is worth mentioning that the number of measurement values $M^{\prime}$ is much less than $M$. Obviously, the $m$-th measurement can be expressed as:

$$
I_{n m}^{C S}=\sum_{i=1}^{M} c_{m i} I_{n}\left(\theta_{i}\right) .
$$

Let $\mathbf{I}_{m}^{C S}=\left\{I_{1 m}^{C S}, I_{2 m}^{C S}, \cdots, I_{N m}^{C S}\right\}$, then we have:

$$
\begin{gathered}
\mathbf{Z I}_{m}^{C S}=\mathbf{Z}\left(\begin{array}{c}
\mathrm{I}_{1 m}^{C S} \\
\mathbf{I}_{2 m}^{C S} \\
\vdots \\
\mathbf{I}_{N m}^{C S}
\end{array}\right)=\mathbf{Z}\left(\begin{array}{c}
\sum_{i=1}^{M} c_{m i} I_{1}\left(\theta_{i}\right) \\
\sum_{i=1}^{M} c_{m i} I_{2}\left(\theta_{i}\right) \\
\vdots \\
\sum_{i=1}^{M} c_{m i} I_{N}\left(\theta_{i}\right)
\end{array}\right) \\
=\sum_{i=1}^{M} c_{m i} \mathbf{Z I}\left(\theta_{i}\right)=\sum_{i=1}^{M} c_{m i} \mathbf{V}\left(\theta_{i}\right) .
\end{gathered}
$$

The right hand side of (9) is a random superposition of $M$ right-hand-side vectors related to different incident angles and the weights are the $m$-th row elements of $\phi$. So the $M^{\prime}$ measurement values of each current coefficient can be obtained by changing the subscript $\mathrm{m}$ in (9) from 1 to $M^{\prime}$. In such a manner, the number of equations to be solved can be greatly reduced.

According to the theory of CS [27], if the unknown vector $\left\{I_{n}\left(\theta_{1}\right), I_{n}\left(\theta_{2}\right), \ldots, I_{n}\left(\theta_{M}\right)\right\}$ is compressible in terms of a orthonormal basis $\Psi$, i.e.,

$$
\left(\begin{array}{c}
I_{n}\left(\theta_{1}\right) \\
I_{n}\left(\theta_{2}\right) \\
\vdots \\
\vdots \\
I_{n}\left(\theta_{M}\right)
\end{array}\right)=\Psi \omega
$$

where $\Psi$ is a $M \times M$ matrix, $\omega=\left\{\omega_{1}, \omega_{2}, \cdots, \omega_{M}\right\}$ has just a few of large coefficients and many small coefficients. Substitute (10) into (7), $\omega$ can be obtained by solving the following matrix equation:

$$
\left(\begin{array}{c}
I_{n 1}^{C S} \\
I_{n 2}^{C S} \\
\vdots \\
I_{n M^{\prime}}^{C S}
\end{array}\right)=\phi \Psi \omega=\Phi \omega,
$$

where $\Phi=\phi \Psi$ is a $M \times M$ sensing matrix. $M^{\prime}$ is much smaller than $M$. Hence, (11) is an underdetermined equation which is a nondeterministic polynomial time (NP)-hard problem. A reconstruction algorithm is required to recover $\omega$ from $M^{\prime}$ measurements. After $\omega$ is solved, we can adopt (10) to obtain the original current coefficient vector.

\section{Bayesian compressive sensing method}

To solve (11), the reconstruction algorithm in [20] is the orthogonal matching pursuit (OMP) algorithm [21]. However, the OMP algorithm is a greedy algorithm and it frequently converges to local optimal. Moreover, the number of measurement cannot be predefined adaptively and one has to try several times to find the optimized values for the number of measurements.

In the Bayesian compressive sensing method, the solution of the NP-hard problem in (11) can be rewritten into the following form:

$$
\mathbf{t}=\Phi \omega+\varepsilon,
$$

where $\mathbf{t}$ is the vector of measurement values, $\boldsymbol{\varepsilon}$ is the expansion error and it is assumed to be zero-mean Gaussian distribution with variance $\sigma^{2}$. Then the vector t obeys a multivariate Gaussian distribution,

$$
p\left(\mathbf{t} \mid \omega, \sigma^{2}\right)=\left(2 \pi \sigma^{2}\right)^{-\frac{M^{\prime}}{2}} \exp \left(-\frac{1}{2 \sigma^{2}}\|\mathbf{t}-\mathbf{\Phi} \omega\|^{2}\right) .
$$

A zero-mean Gaussian prior is defined over $\omega$ :

$$
p(\boldsymbol{\omega} \mid \mathbf{a})=\prod_{i=1}^{M} N\left(\omega_{i} \mid 0, a_{i}^{-1}\right),
$$

where $\mathbf{a}=\left[a_{1}, a_{2}, \cdots, a_{M}\right]^{T}$ is a vector of $M$ independent hyperparameters and $a_{i}$ is the precision (reciprocal of variance) of a Gaussian distribution.

For the fixed values of hyperparameters controlling the prior, the posterior probability density of the weights can be obtained:

$$
p\left(\boldsymbol{\omega} \mid \mathbf{t} ; \mathbf{a}, \sigma^{2}\right)=N(\boldsymbol{\omega} \mid \boldsymbol{\mu}, \boldsymbol{\Sigma}),
$$

where its mean and covariance are:

$$
\begin{gathered}
\boldsymbol{\mu}=\sigma^{-2} \boldsymbol{\Sigma} \boldsymbol{\Phi}^{T} \mathbf{t}, \\
\boldsymbol{\Sigma}=\left(\mathbf{A}+\sigma^{-2} \boldsymbol{\Phi}^{T} \boldsymbol{\Phi}\right)^{-1},
\end{gathered}
$$

with $\mathbf{A}=\operatorname{diag}\left(a_{1}, a_{2}, \cdots, a_{M}\right)$.

According to (13) and (14), the marginal distribution of $\mathbf{t}$ can be computed as:

$$
p\left(\mathbf{t} \mid \mathbf{a}, \sigma^{2}\right)=(2 \pi)^{-M^{\prime} / 2}|\mathbf{C}|^{-1 / 2} \exp \left(-\frac{1}{2} \mathbf{t}^{\mathrm{T}} \mathbf{C}^{-1} \mathbf{t}\right),
$$

where

$$
\mathbf{C}=\sigma^{2} \mathbf{I}+\boldsymbol{\Phi} \mathbf{A}^{-1} \boldsymbol{\Phi}^{T} .
$$

In the sparse Bayesian learning method, the maximization of $p\left(\mathbf{t} \mid \mathbf{a}, \sigma^{2}\right)$ is termed as type-II maximum likelihood method. The hyperparameters a and $\sigma^{-2}$ are estimated through computing the derivatives of (18) with respect to a and $\sigma^{-2}$ :

$$
\begin{gathered}
a_{i}^{\text {new }}=\frac{\gamma_{i}}{\mu_{i}^{2}}, \\
\left(\sigma^{2}\right)^{\text {new }}=\frac{\|\mathbf{t}-\boldsymbol{\Phi} \boldsymbol{\mu}\|^{2}}{M^{\prime}-\sum_{i=1}^{M} \gamma_{i}},
\end{gathered}
$$


where $\gamma_{i}=1-a_{i} \sum_{i i}, \sum_{i i}$ is the $i$ th diagonal element of the covariance in (17).

The formulas (16) and (17) coupled with (20) and (21) lead to an iterative learning process, which updates the corresponding quantity until a convergence criterion is satisfied. Many elements of a tend to infinity during the iteration with the consequence that $\mu$ contains very few non-zero elements. After the convergence of the iterative learning process, $\boldsymbol{\mu}$ is used to approximate $\boldsymbol{\omega}$.

Since the diagonal elements of the covariance matrix $\boldsymbol{\Sigma}$ correspond to the variance of each element in $\boldsymbol{\omega}$, they provide error bars on the accuracy of $\boldsymbol{\omega}$. When the number of measurement is sufficiently large, the variance of each element in $\boldsymbol{\omega}$ should be small. If the diagonal elements of the covariance matrix $\boldsymbol{\Sigma}$ is $\Sigma_{i i}, i=1,2, \cdots, M$, the number of measurement times $M^{\prime}$ is enough when,

$$
\frac{\Sigma_{11}+\Sigma_{22}+\cdots+\Sigma_{M M}}{M}<\delta,
$$

where $\delta$ is a small value and $\delta=10^{-3}$ in this paper. If (22) is not satisfied, more measurement will be added. In such a manner, the proposed method can adaptively determine the number of measurement.

\section{NUMERICAL RESULTS}

The effectiveness and accuracy of the proposed method are demonstrated through several numerical results. All results are generated on a personal PC with $2.83 \mathrm{GHz} \mathrm{CPU}$ and $8 \mathrm{GHz}$ RAM. The flexible general minimal residual (FGMRES) algorithm is adopted to solve the matrix equation and the iteration process is terminated when the 2-norm residual error is reduced by $10^{-3}$. Multilevel fast multipole method (MLFMM) is utilized to accelerate the matrix vector product process.

\section{A. Almond}

The NASA almond model is analyzed as the first example as shown in Fig. 2 [28]. It is discretized with 3290 triangular patches with 4935 unknowns. The tip of the almond points to the $\mathrm{x}$-axis. The elevation angle of the incident wave is fixed to be $90^{\circ}$, while the aspect angle ranges from $0^{\circ}$ to $63^{\circ}$ with a $1^{\circ}$ increment. Since the basis matrix $\Psi$ has an important effect on the measurement number, we compare three different bases in this example including Hermite basis, discrete cosine transformation (DCT) basis and Haar wavelet basis. All these basis are adopted to analyze the monostatic scattering problem and their results are compared with the result of MLFMM. The real parts of current coefficients at a randomly chosen edge under different incident angles are shown in Fig. 3. It can be observed that the results obtained by Hermite and DCT basis agree well with that of MLFMM. The result obtained by using Haar basis is comparable to that of MLFMM.
The numbers in the brackets means the corresponding measurement times. Note that the measurement number corresponds to the number of MoM solutions. Obviously, the measurement times after adopting Hermite basis achieve its minimum. So the basis function is fixed to be the Hermite basis in the following two examples. Figure 4 demonstrates the current magnitude distributions obtained by MLFMM and the proposed method with different basis. Good agreement can be achieved.

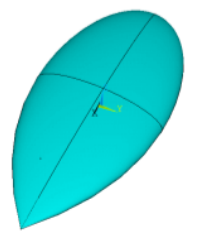

Fig. 2. Almond model.

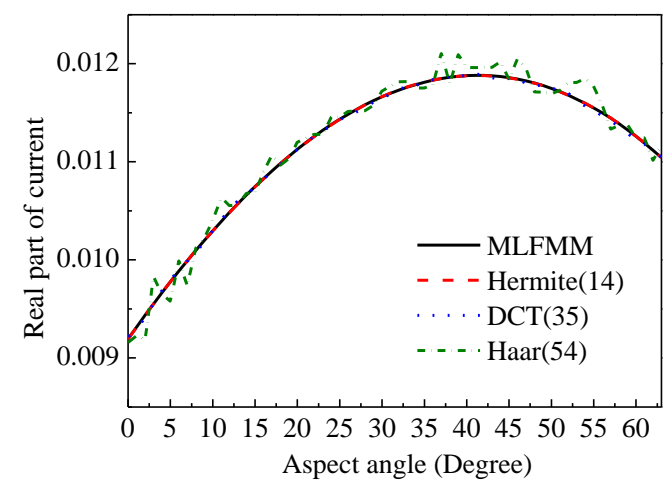

Fig. 3. Real parts of current coefficients at a randomly chosen edge under different incident angles obtained by using three kinds of basis. The numbers in the brackets represent the number of measurement.

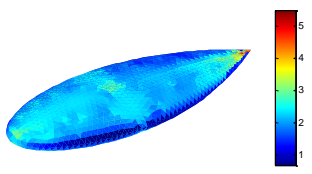

(a)

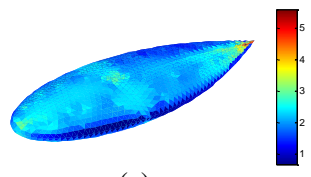

(c)

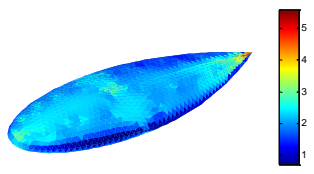

(b)

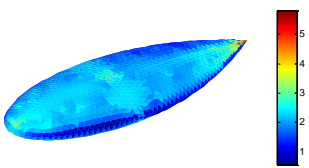

(d)
Fig. 4. The current magnitude distributions obtained by MLFMM and the proposed method with different basis when the aspect angle is $0^{\circ}$ : (a) MLFMM, (b) Hermite, (c) DCT, and (d) Haar.

\section{B. Missile model}

A PEC missile model as shown in Fig. 5 is analyzed as the second example. The model is created based on 
the picture of Tomahawk missile in Wikipedia [29]. The maximum size in the $\mathrm{x}, \mathrm{y}$ and $\mathrm{z}$ directions are $1.4 \mathrm{~m}$, $0.62 \mathrm{~m}$ and $0.25 \mathrm{~m}$. It is discretized into 6792 unknowns at $1.5 \mathrm{GHz}$. The warhead is towards the positive direction of $\mathrm{x}$-axis. The elevation angle of the incident wave is fixed to be $90^{\circ}$ while the aspect angle ranges from $0^{\circ}$ to $180^{\circ}$ with $0.5^{\circ}$ increment. Both the proposed method (BCS) and the method in [20] (CS_OMP) are adopted to analyze the monostatic scattering problem and their results are compared with that of MLFMM as shown in Fig. 6. Table 1 lists the measurement number and CPU time for different methods. The number of measurement of the proposed method is determined to be 63 adaptively, and the result match well with that of MLFMM. Since the CS_OMP method cannot determine the number of measurement, we try several different measurement number and select the smallest one giving the similar level of accuracy with the proposed method. The measurement number determined in such a manner for CS_OMP method is 71. Although the measurement time of the proposed method is less than the CS_OMP method, their CPU time is similar since the computational cost of BCS algorithm is larger than OMP algorithm.

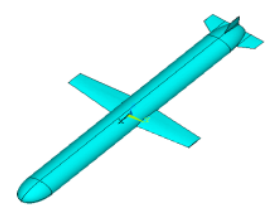

Fig. 5. Missile model.

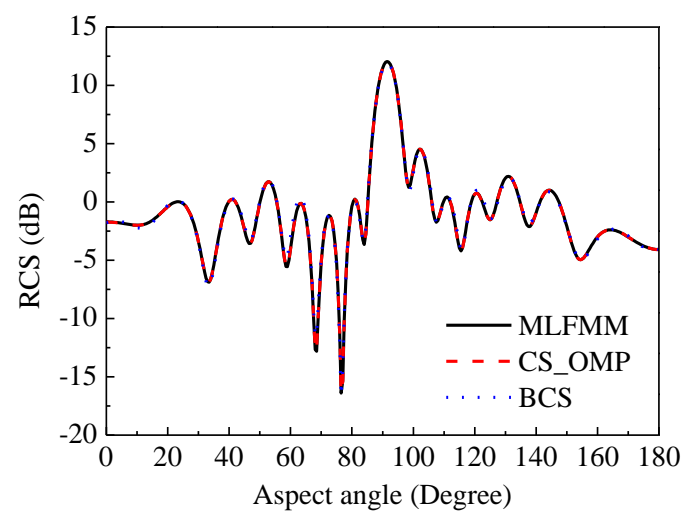

Fig. 6. Monostatic RCS of the missile model obtained by MLFMM, CS_OMP and BCS.

Table 1: Measurement number and CPU time of three kinds of methods for the missile model

\begin{tabular}{c|c|c}
\hline Method & Measurement Number & CPU Time (s) \\
\hline MLFMM & 360 & 5527 \\
\hline CS_OMP & 71 & 1272 \\
\hline BCS & 63 & 1295 \\
\hline
\end{tabular}

\section{Aircraft model}

A scaled aircraft model shown in Fig. 7 is analyzed as the third example. The model is created based on the picture of F15 fighter plane in Wikipedia [30]. The maximum size in the $\mathrm{x}, \mathrm{y}$ and $\mathrm{z}$ directions are $1.9 \mathrm{~m}$, $1.2 \mathrm{~m}$ and $0.4 \mathrm{~m}$. It is discretized into 6741 unknowns at $600 \mathrm{MHz}$. The nose of the aircraft is towards the positive direction of $\mathrm{x}$-axis. The elevation angle of the incident wave is fixed to be $90^{\circ}$ while the aspect angle ranges from $0^{\circ}$ to $360^{\circ}$ with $1^{\circ}$ increment. Figure 8 demonstrates the monostatic RCS computed by the MLFMM, CS_OMP and BCS method. Table 2 lists the measurement number and CPU time for different methods. Similar conclusions can be drawn with the second numerical example.

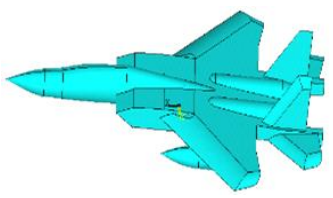

Fig. 7. Aircraft model.

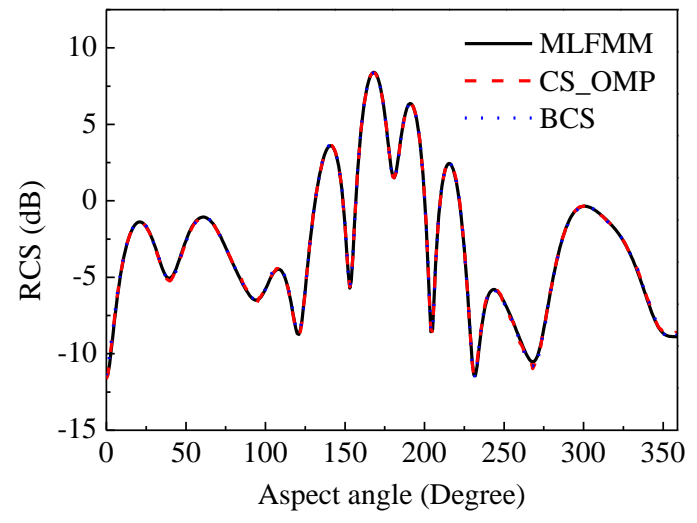

Fig. 8. Monostatic RCS of the aircraft model obtained by MLFMM, CS_OMP and BCS.

Table 2: Measurement number and CPU time of three kinds of methods for the aircraft model

\begin{tabular}{c|c|c}
\hline Method & Measurement Number & CPU Time (s) \\
\hline MLFMM & 360 & 15817 \\
\hline CS_OMP & 80 & 3534 \\
\hline BCS & 69 & 3505 \\
\hline
\end{tabular}

\section{CONCLUSION}

The Bayesian compressive sensing method is applied to the fast monostatic scattering analysis. Compared with the traditional CS based method, the proposed method adopts the Bayesian framework and can adaptively determine the number of compressivesensing measurements. Moreover, the proposed method 
needs less measurements than OMP method with the similar level of accuracy.

\section{ACKNOWLEDGMENT}

This work is supported by the Fundamental Research Funds for the Central Universities JB160218, XJS16048 and the NSFC 61301069.

\section{REFERENCES}

[1] R. F. Harrington, Field Computation by Moment Methods. Oxford University Press, 1996.

[2] J. J. Bai, G. Zhang, L. X. Wang, and T. H. Wang, "Uncertainty analysis in EMC simulation based on improved method of moments," Appl. Comput. Electromagn. Soc. J., vol. 31, no. 1, pp. 66-71, 2016.

[3] G. Apaydin and L. Sevgi, "A novel wedge diffraction modeling using method of moments (MoM)," Appl. Comput. Electromagn. Soc. J., vol. 30, no. 10, pp. 1053-1058, 2015.

[4] J. M. Song, C. C. Lu, and W. C. Chew, "Multilevel fast multipole algorithm for electromagnetic scattering by large complex objects," IEEE Trans. Antennas Propagat., vol. 45, no. 10, pp. 14881493, 1997.

[5] J. Song and W. C. Chew. "The fast Illinois solver code: Requirements and scaling properties," IEEE Comput. Sci. Eng., vol. 5, no. 3, pp. 19-23, 1998.

[6] J. Song and W. C. Chew., "Multilevel fastmultipole algorithm for solving combined field integral equations of electromagnetic scattering," Microw. Opt. Technol. Lett., vol. 10, no. 1, pp. 1419, 1995.

[7] E. Bleszynski, M. Bleszynski, and T. Jaroszewicz, "AIM: Adaptive integral method for solving largescale electromagnetic scattering and radiation problems," Radio Science, vol. 31, no. 5, pp. 12251251, 1996.

[8] J. R. Phillips and J. K. White, "A precorrected-FFT method for electrostatic analysis of complicated 3D structures," IEEE Trans. Comput.-Aided Des. Integr. Circuits Syst., vol. 16, no. 10, pp. 10591072, 1997.

[9] S. M. Seo and J. F. Lee, "A fast IE-FFT algorithm for solving PEC scattering problems," IEEE Trans. Magn., vol. 41, no. 5, pp. 1476-1479, 2005.

[10] W. Chai and D. Jiao, "An-matrix-based integralequation solver of reduced complexity and controlled accuracy for solving electrodynamic problems," IEEE Trans. Antennas Propagat., vol. 57, no. 10, pp. 3147-3159, 2009.

[11] K. Zhao, M. N. Vouvakis, and J. F. Lee, "The adaptive cross approximation algorithm for accelerated method of moments computations of EMC problems," IEEE Trans. Electromagn. Compat., vol. 47, no. 4, pp. 763-773, 2005.
[12] J. M. Rius, J. Parron, A. Heldring, J. M. Tamayo, and E. Ubeda, "Fast iterative solution of integral equations with method of moments and matrix decomposition algorithm-singular value decomposition," IEEE Trans. Antennas Propagat., vol. 56, no. 8, pp. 2314-2324, 2008.

[13] Y. E. Erdemli, J. Gong, C. J. Reddy, and J. L. Volakis, "Fast RCS pattern fill using AWE technique," IEEE Trans. Antennas Propagat., vol. 46, no. 11, pp. 1752-1753, 1998.

[14] M. S. Chen, X. L. Wu, and W. Sha, "Asymptotic waveform evaluation technique based on fast lifting wavelet transform," Appl. Comput. Electromagn. Soc. J., vol. 21, no. 1, pp. 99-104, 2006.

[15] E. K. Miller, "Model-based parameter estimation in electromagnetic III: Applications to EM integral equations," IEEE Antennas Propag. Mag., vol. 40, no. 3, pp. 49-66, 1998.

[16] Z. Peng, M. Stephanson, and J. F. Lee, "Fast computation of angular responses of largescale three-dimensional electromagnetic wave scattering," IEEE Trans. Antennas Propag., vol. 58, no. 9, pp. 3004-3012, 2010.

[17] A. Schröder, H.-D. Brüns, and C. Schuster, "A hybrid approach for rapid computation of twodimensional monostatic radar cross section problems with the multilevel fast multipole algorithm," IEEE Trans. Antennas Propag., vol. 60, no. 12, pp. 6058-6061, 2012.

[18] A. Massa, P. Rocca, and G. Oliveri, "Compressive sensing in electromagnetics - A review," IEEE Antennas Propag. Mag., vol. 57, no. 1, pp. 224238, 2015.

[19] A. F. Morabito, A. R. Laganà, G. Sorbello, and T. Isernia, "Mask-constrained power synthesis of maximally sparse linear arrays through a compressive-sensing-driven strategy," Journal of Electromagnetic Waves and Applications, vol. 29, no. 10, pp. 1384-1396, 2015.

[20] M. S. Chen, F. L. Liu, H. M. Du, and X. L. Wu, "Compressive sensing for fast analysis of wideangle monostatic scattering problems," IEEE Antennas Wirel. Propag. Lett., vol. 10, pp. 12431246, 2011.

[21] J. A. Tropp and A. Gilbert, "Signal recovery from partial information by orthogonal matching pursuit," IEEE Trans. Inf. Theory, vol. 53, no. 12, pp. 4655-4667, 2007.

[22] S. H. Ji, Y. Xue, and L. Carin, "Bayesian compressive sensing," IEEE Trans. Signal Process., vol. 56, no. 6, pp. 2346-2356, 2008.

[23] D. Baron, S. Sarvotham, and R. G. Baraniuk, "Bayesian compressive sensing via belief propagation," IEEE Trans. Signal Process., vol. 58, no. 1, pp. 269-280, 2010.

[24] H. H. Zhang and R. S. Chen, "Coherent processing 
and superresolution technique of multi-band radar data based on fast sparse Bayesian learning algorithm," IEEE Trans. Antennas Propag., vol. 62, no. 12, pp. 6217-6227, 2014.

[25] M. Carlin, P. Rocca, G. Oliveri, F. Viani, and A. Massa, "Directions-of-arrival estimation through Bayesian compressive sensing strategies," IEEE Trans. Antennas Propag., vol. 61, no. 7, pp. 38283838, 2013.

[26] S. M. Rao, D. R. Wilton, and A. W. Glisson, "Electromagnetic scattering by surfaces of arbitrary shape," IEEE Trans. Antennas Propagat, vol. 30, no. 3, pp. 409-418, 1982.

[27] E. J. Candes and M. B. Wakin, "An introduction to compressive sampling," IEEE Signal Process. Mag., vol. 25, no. 2, pp. 21-30, 2008.

[28] A. C. Woo, H. T. G. Wang, M. J. Schuh, and M. L. Sanders, "Benchmark radar targets for the validation of computational electromagnetics programs," IEEE Trans. Antennas Propagat, vol. 35, no.1, pp. 84-89, 1993.

[29] https://en.wikipedia.org/wiki/Tomahawk_(missile)

[30] https://en.wikipedia.org/wiki/McDonnell_Douglas _F-15_Eagle

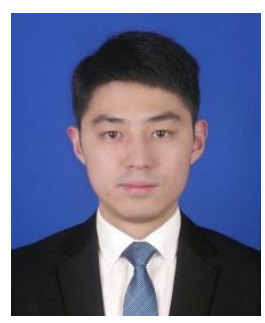

Huan Huan Zhang received the Ph.D. degree in Electromagnetic Fields and Microwave Technology from Nanjing University of Science and Technology in 2015. He was a Postdoctoral Research Fellow with the Center of Electromagnetics and Optics, the University of Hong Kong, Hong Kong, from 2015 to 2016 . He is currently a Lecturer with The School of Electronic Engineering, Xidian University, Xi'an, China.

Zhang serves as the Reviewer of the IEEE Transactions on Antenna and Propagation, Communications in Computational Physics, IET Radar, Sonar \& Navigation, etc. His current research interests include computational electromagnetics, IC signal integrity, EMC/EMI and radar signal processing.

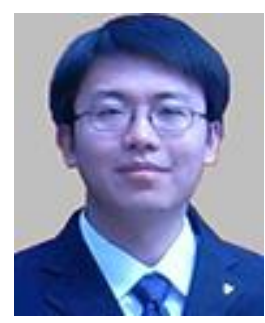

Xun Wang Zhao received the B.S. and Ph.D. degrees from Xidian University, Xi'an, China, in 2004, and 2008, respectively. He joined Xidian University as a Faculty Member in 2008. As principal Investigator, he is doing or has completed some projects including

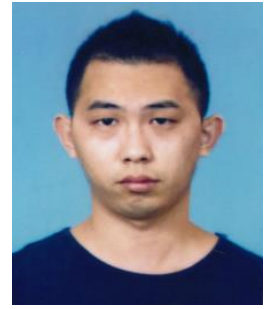

Zhong Chao Lin received the B.S. degree from Xidian University, Xi'an, China, in 2011, and is currently working toward the Ph.D. degree at the School of Electronic and Engineering, Xidian University, Xi'an, China. His current research interests is computational electromagnetic.

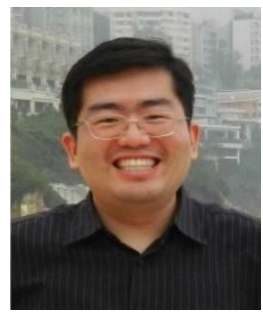

Wei E. I. Sha has co-authored two books respectively on wavelet theory and finite-difference time-domain method. He has published 72 peerreviewed journal papers included in Web of Science Core Collection. He also contributed four book chapters at Springer, CRC Press and InTech Publishers and 13 invited talks at international conferences. Sha is an IEEE Member and an OSA Member. He has served as the Onsite Award Committee, Technical Program Committee or Session Chair of several international conferences including ICCEM 2017/2016, PIERS 2016, IMWS-AMP 2015, EICT 2015, ICSPS 2011, etc. He has been serving as the Invited Referees of IEEE, OSA, AIP, APS and Nature Publishing Group journals. He also served as the Book Proposal Reviewers for CRC Press and Bentham Science publishers.

He received the Second Prize and First Prize of National Postgraduates Mathematical Contest in Modeling, respectively in 2006 and 2007. He was awarded Chinese Youth Science and Technology Innovation Prize in 2007. He and his collaborators received Research Output Prize at the University of Hong Kong in 2013. In 2014, he was awarded Outstanding Reviewer of Journal of Computational Physics. In 2015, he was awarded Second Prize of Natural Science from Anhui Province Government, China. Sha also received three Best Student Paper Prizes and one Young Scientist Award with his students.

He engages in theoretical and computational research in electromagnetics and optics, focusing on the multiphysics and interdisciplinary research. The research topics are inspired by applications in several areas including solar energy, microwave/optical communication, sensing/detection, and quantum information. His research involves fundamental and applied aspects in plasmonics, emerging photovoltaics, metasurfaces, quantum electrodynamics, and computational electromagnetics. project of NSFC. 